\title{
LA EVOLUCIÓN ECONÓMICA DE LA REGIÓN DE LOS RÍOS DESDE SU CREACIÓN, A PARTIR DE UN ANÁLISIS INPUT-OUTPUT ${ }^{\bullet}$
}

\author{
JUAN CARLOS MIRANDA CASTILLO
}

Instituto de Estadística de la Facultad de Ciencias Económicas y Administrativas, UNIVERSIDAD AUSTRAL. CHILE.

e-mail: jmiranda@uach.cl

CARMEN RAMOS CARVAJAL (Autor para correspondencia)

Departamento de Economía Aplicada, UNVERSIDAD DE OVIEDO.ESPAÑA.

e-mail: cramos@uniovi.es

RENÉ REYES IRIGOYEN

Departamento de Ciencias Administrativas y Económicas, UNIVERSIDAD DE LOS LAGOS. CHILE.

e-mail: rreyes@ulagos.cl

FELIPE ACUM BOASSI

Departamento de Ciencias Administrativas y Económicas, UNIVERSIDAD DE LOS LAGOS.CHILE.

e-mail: felipe.acum@gmail.com

LUIS HERNÁN VIDAL VIDAL

Instituto de Ingeniería Industrial y Sistemas. Facultad de Ciencias de la Ingeniería, UNIVERSIDAD AUSTRAL.CHILE.

e-mail: lvidal@uach.cl

\section{RESUMEN}

En este trabajo se estudia la economía de la Región de los Ríos (Chile) y se analiza su evolución en el período 2007-2016. Para llevar a cabo dicho estudio se ha aplicado el análisis insumo-producto, en concreto, se ha realizado un análisis de la estructura productiva sectorial y clasificado a los sectores mediante los coeficientes de Chenery y Watanabe y de Rasmussen. Asimismo, se han determinado las jerarquías sectoriales de acuerdo a la construcción de la matriz producto de multiplicadores (MPM). Por otra parte, también se han analizado la complejidad de la economía regional, a partir de la determinación de la longitud media de propagación (APL)y el cambio estructural y su descomposición tanto en las fuentes de dicho cambio (demanda final, tecnología e interacción de ambas) como por su procedencia (del propio sector o del resto de sectores).

Palabras clave: matriz insumo-producto, encadenamiento productivo, Región de Los Ríos, cambio estructural.

\section{The economic evolution of the La Region de los Ríos since its creation from an Input- Output analysis}

\begin{abstract}
In this paper the economy of the Region de Los Ríos (Chile) is studied and its evolution is analyzed in the period 2007-2016. To carry out this study, the input-output analysis has been applied. Specifically, an analysis of the sectorial productive structure has been made and classified to the sectors through the coefficients of Chenery and Watanabe and Rasmussen. Likewise, the sectoral hierarchies have been determined according to the construction of the product matrix of multipliers (MPM). On the other hand, the complexity of the regional economy has also been analyzed, from the determination of the average length of propagation (APL) and the structural change and its decomposition in the sources of this change (final demand, technology and interaction of both) and by their origin (of the sector itself or of the other sectors).
\end{abstract}

Keywords: Input-Output Matrix, Productive Linkage, Los Ríos Region, Structural Change

Clasificación JEL: C67, D57, R11.

\footnotetext{
- Actividad del núcleo de investigación RiNA financiada por el Proyecto DID-UACh (2015-2018) y el Proyecto meso regional transforma turismo de CORFO, financiado por InnovaChile (2016-2018).

Artículo recibido el 2 de octubre de 2018 y aceptado el 6 de diciembre de 2018

Artículo disponible en versión electrónica en la página www.revista-eea.net

ISSN 1697-5731 (online) - ISSN 1133-3197 (print)
} 


\section{INTRODUCCIÓN}

En la actualidad, las regiones en Chile se mueven dentro de un escenario de descentralización; en momentos, como el presente, en que los territorios reclaman cada vez más voz y voto, atribuciones y recursos, se precisan herramientas cuantitativas versátiles que permitan tanto el conocimiento de la economía actual como su proyección en situaciones en las que se produzcan cambios en determinados parámetros.

El desarrollo económico y territorial de una región, especialmente las que, como la de referencia, están en proceso de Reforma Constitucional ${ }^{\circ}$, permitirá una descentralización política, administrativa y fiscal efectiva. Dicho desarrollo requiere que se disponga de más información y de un bagaje de técnicas que permitan su análisis para coadyuvar a la toma de decisiones por parte de las autoridades competentes. Estas decisiones serán más adecuadas y oportunas, en tanto en cuanto, empleen instrumentos metodológicos flexibles que permitan el conocimiento de la economía.

El análisis insumo-producto es una herramienta de gran utilidad en los estudios económicos, ya que una matriz insumo-producto (MIP) contiene un amplio volumen de información referente a las transacciones entre los distintos sectores, a la demanda final y a los insumos primarios, así como a las relaciones con el resto del mundo que pueden ser cuantificadas a partir de las importaciones y exportaciones; por todo ello, permite un conocimiento detallado de una región (o nación). Una de las principales ventajas de la utilización de una MIP es que posibilita llevar a cabo tanto un análisis profundo de la estructura económica de una región, como efectuar proyecciones y simulaciones de la misma de una manera relativamente sencilla; por ello, su explotación permite obtener un conocimiento detallado de la economía regional cierto y posible. Cierto, dado que permite analizar la economía real, y posible, al permitir realizar proyecciones y simulaciones de dicha economía, que faculten a la autoridad competente para tomar decisiones correctas e incluso adelantarse a determinados acontecimientos.

La estructura productiva de un territorio está constituida, en términos generales, por los sectores que la componen y por las relaciones establecidas entre los mismos. El transcurso del tiempo o la ocurrencia de perturbaciones económicas o sociales pueden hacer que la importancia de los sectores o sus interrelaciones varíen. Así, ramas con menos peso en un momento dado pueden ver crecer su relevancia y viceversa (Hewings, et al, 1988, Thakur, 2011, Thakur y Alvayay, 2012). Dado que la Región de los Ríos es de relativamente reciente creación puede esperarse que su estructura esté sujeta a una mayor variabilidad que la de otras economías con un mayor grado de madurez. Siguiendo a Hewings et al (1988) presentamos un gráfico que muestra de manera estilizada los cambios que se producen en una estructura productiva desde su nacimiento hasta su madurez.

A medida que una economía evoluciona desde una etapa inicial a otra con mayor grado de madurez, las interrelaciones y los vínculos entre sectores se complejizan. Desde la etapa inicial, las interdependencias de la economía aumentan gradualmente para, posteriormente, crecer a un ritmo más rápido, lo que lleva a una fuerte interacción entre los sectores. En una etapa madura de desarrollo se ralentiza el ritmo de las interacciones entre industrias, lo que lleva a la posibilidad de un declive posterior en las interrelaciones, conocido como el proceso de "hollowingout".

\footnotetext{
- Dicha reforma constitucional se rige por la Ley 20.990 de 5 de Enero de 2017.
} 
Figura 1.

Evolución de la complejidad de una economía

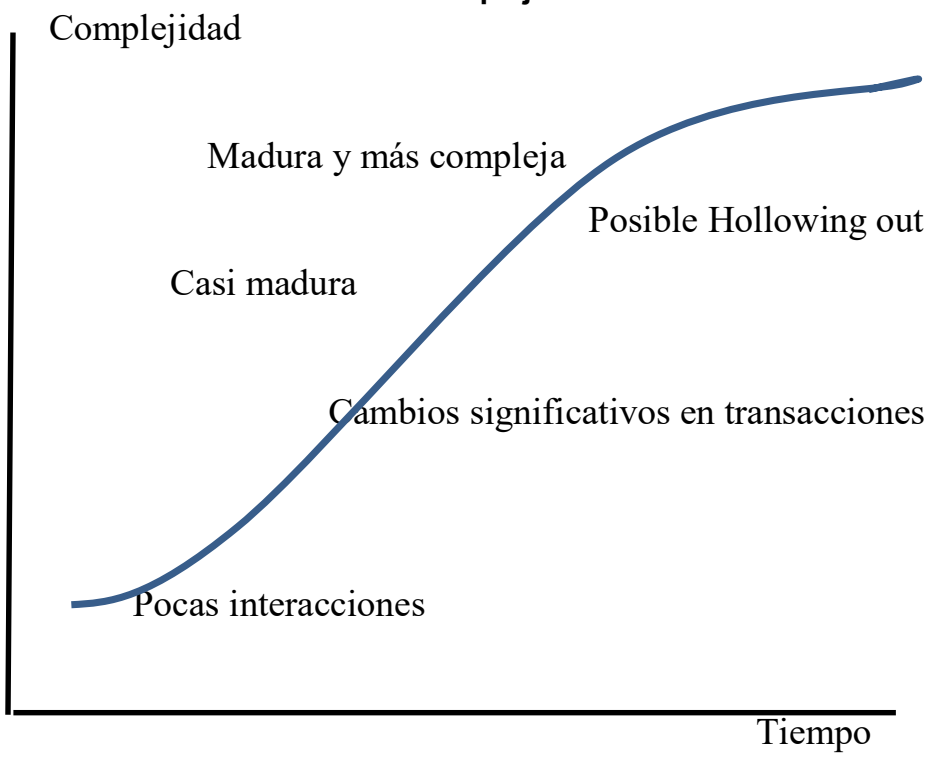

Fuente: Hewings et al (1988).

Los objetivos de este trabajo son fundamentalmente dos, por una parte, se analizará la economía de la Región de los Ríos en la actualidad y, por otra, se intentará conocer cómo ha evolucionado. El procedimiento para alcanzar estos objetivos se fundamenta en la utilizaciónde dos grupos de técnicas dentro de la metodología insumo-producto:

a. Aplicación de técnicas que permiten analizar la estructura productiva en cada uno de los años considerados $\mathrm{y}$, posteriormente, efectuar su comparación. De esta forma se podrá tener conocimiento de la economía regional "actual", además de poder determinar los efectos del transcurso del tiempo.

b. Aplicación de técnicas de descomposición de cambio estructural, las cuales permitirán evaluar la magnitud de dicho cambio sobre ciertas variables económicas.

Este trabajo se estructura de la siguiente manera: en el apartado segundo se realizará una breve referencia a algunos conceptos básicos para la comprensión del modelo insumo-producto, así como una descripción de las técnicas que serán empleadas en este estudio. En el apartado tercero se comentará cómo se han llevado a cabo las estimaciones de la MIP de la Región de los Ríos y del PIB. En el apartado cuarto se llevará a cabo el análisis de la estructura económica de la Región en el año 2016, así como su comparación con la de 2007. En el apartado quinto se efectuarán unas breves conclusiones del estudio realizado.

\section{METODOLOGÍA DEL ANÁLISIS}

Como ya se ha señalado, la metodología que va a ser aplicada en este trabajo es el análisis insumoproducto, el cual permite efectuar estudios sobre la estructura productiva de una región o nación de una manera relativamente sencilla, pero muy clarificadora a la vez.

En este apartado vamos a presentar brevemente algunos de los conceptos básicos que nos permitirán el desarrollo de este trabajo.

\subsection{Conceptos básicos del análisis insumo-producto}

Sea el denominado modelo de cantidades (Modelo de Leontief): 
$x=X_{i}+y$

Tal que x representa el vector de producción total, i es un vector de unos, $\mathrm{X}$ es la matriz de producción intermedia e y la demanda final.

Se definen los coeficientes técnicos aij, que se suponen constantes, como:

$a_{i j}=\frac{X_{i j}}{x_{j}}$

Los cuales pueden ser interpretados comola proporción de bienes y servicios comprados por el sector $\mathrm{j}$ al sector i respecto del total de la producción del sector j. Considerando las ecuaciones (1) y (2) se tiene que $\mathrm{x}=\mathrm{Ax}+\mathrm{y}$, donde la matriz A tiene por elementos los coeficientes técnicos. Si se opera convenientemente, se obtiene:

$$
x=(I-A)^{-1} y
$$

La expresión (I-A)-1 representa a la inversa de Leontief. Un elemento genérico (i,j) de esta matriz se interpreta como las necesidades directas e indirectas del bien i, necesarias para satisfacer la demanda final del j.

Análogamente, el modelo de precios de Ghosh (1958) toma la siguiente forma: $\mathrm{x}^{\prime}=\mathrm{x}^{\prime} \mathrm{B}+\mathrm{w}^{\prime}$, donde $\mathrm{B}$ representa a la matriz de distribución con elementos $\left\{\frac{x_{i j}}{x_{i}}\right\} y$ w es la matriz de inputs primarios. Asimismo, también puede presentarse la matriz inversa de Ghosh, (I-B)-1, que permite derivar la expresión siguiente $\mathrm{x}^{\prime}=\mathrm{w}^{\prime}(\mathrm{I}-\mathrm{B})-1$, simétrica de (3).

Sobre este modelo (Modelo de Ghosh), su interpretación, limitaciones y conexión con el modelo de Leontief pueden verse, entre otros, los trabajos de Oosterhaven (1988, 1996, 2012), Rose y Allison (1889) y Dietzenbacher (1997).

\subsection{Indicadores de la estructura productiva}

A fines de la década de los años 50 del pasado siglo se planteó una discusión sobre los factores que influyen en el crecimiento económico, así, el modelo de Harrod-Domar,que sostenía que el crecimiento dependía, casi exclusivamente, de la relación capital-producto y de la disponibilidad del capital, fue cuestionado por Hirschman (Franco y Ramírez, 2005 y Pino e Illanes, 2002),el cualmantenía que "el desarrollo depende no tanto de encontrar las combinaciones óptimas para los recursos y factores de producción, como de provocar e incorporar para el desarrollo, recursos y capacidades que están ocultos, diseminados o mal utilizados" (Hirschman, 1958). Este autor introduce el concepto de "eslabonamiento", el cualrequiere valorar las decisiones de invertir tanto considerando su efecto inmediato sobre el output como los estímulos adicionales que dichas decisiones ejercen. Distingue dos tipos de eslabonamiento: hacia atrás (BL) y hacia delante (FL). El primero, mide la capacidad de una actividad económica para "arrastrar" al conjunto de sectores, al demandarlesproductos que serán utilizados por ella como insumos en la fabricación de su bien final. Por su parte, el eslabonamiento hacia delante, muestra la capacidad de un sector económico de "empujar" a otras ramas, a las que ofertaproductos que serán utilizados por ellas como insumos.

\subsubsection{Enfoque clásico: Coeficientes de Chenery y Watanabe (1958) y Rasmussen (1956)}

Chenery y Watanabe (1958) proponen unos coeficientes, a partir de los cuales se determinan los eslabonamientos directos existentes entre los distintos sectores de una economía. Consideran como más relevantes a aquellos cuyos efectos, en términos relativos, son mayores que la unidad. Dichos indicadores se determinarán a partir de la formulación siguiente:

$$
\mathrm{BL}_{R}^{R}=\frac{\mathrm{ni} A}{\mathrm{i}^{\prime} \mathrm{Ai}} \text { y } \mathrm{FL}_{\mathrm{R}}^{\mathrm{CH}-\mathrm{W}}=\frac{\mathrm{nBi}}{\left(\mathrm{i}^{\prime} \mathrm{Bi}\right)}
$$


Donde A es la matriz de coeficientes técnicos, B la matriz de coeficientes de distribución, i es una matriz columna con valores iguales a uno y n el número de sectores considerados.

Los coeficientes de Rasmussen normalizados, cuantifican los encadenamientos totales (directos e indirectos) y se obtienen de la aplicación de las expresiones siguientes:

$$
\mathrm{BL}_{R}^{R}=\frac{\mathrm{ni}^{\prime}(\mathrm{I}-\mathrm{A})^{-1}}{\mathrm{i}^{\prime}(\mathrm{I}-\mathrm{A})^{-1} \mathrm{i}} \text { y } \mathrm{FL}_{R}^{R}=\frac{\mathrm{n}(\mathrm{I}-\mathrm{B})^{-1} \mathrm{i}}{\mathrm{i}^{\prime}(\mathrm{I}-\mathrm{B})^{-1} \mathrm{i}}
$$

Donde (I-A)-1 representa a la inversa de Leontief y (I-B)-1 es la inversa de Ghosh.

Estos indicadores, a pesar de sus limitaciones, son frecuentemente utilizados por su fácil implementación y clara interpretación. Así, por referirnos a publicaciones actuales, podemos señalar algunos trabajos aplicados, como elde Morrone (2017), en el que se comparan dichos índices con otros métodos; Ramos y otros (2017), los cuales analizan la economía colombiana; Freytagy Fricke (2017), que estudian los servicios financieros de Nigeria y Kenia o Kelly y otros (2016), que analizan las infraestructuras en Reino Unido, entre muchos otros.

A partir de los encadenamientos hacia atrás y hacia delante puede llevarse a cabo la conocida clasificación en sectores clave para la economía, que son los que presentan fuertes encadenamientos hacia delante y hacia atrás; sectores impulsores que son los que muestran elevados eslabonamientos hacia atrás y no hacia delante; sectores base con altos encadenamientos hacia delante y no hacia atrás y sectores independientes con bajos eslabonamientos tanto hacia atrás como hacia delante.

\subsubsection{Análisis de las jerarquías sectoriales}

En este mismo sentido, ladenominada matriz producto de multiplicadores (MPM), propuesta por Sonis et al (1997), permite una caracterización similar a la debida a Rasmussen, dado que considera también ambos tipos de eslabonamientos. Además, recoge el efecto global de la interrelación de una rama con todas las demás y permite una representación clara de las jerarquías sectoriales. En concreto, dicha matriz MPM se define como el producto de un vector columna cuyos elementos son los encadenamientos hacia delante ${ }^{\bullet}$ sectoriales, por un vector fila de encadenamientos hacia atrás, normalizado por la suma de todos los elementos de la matriz de inversa de Leontief. La matriz MPMtiene una estructura interconectada, de manera que se puede identificar el cruce principal (una fila y una columna) como el mayor efecto multiplicador en la economía. Al ordenar los cruces de mayor a menor se obtiene un panorama económico descendente, el cual puede ser usado para determinar la jerarquía de los sectores de acuerdo a valores de los multiplicadores. La matriz MPM se puede definir como:

$$
\mathrm{MPM}=\frac{1}{\mathrm{~V}} \mathrm{~L}_{\mathrm{i} .} \mathrm{L}_{. \mathrm{j}}=\frac{1}{\mathrm{~V}}\left[\begin{array}{c}
\mathrm{l}_{1 .} \\
\vdots \\
\mathrm{l}_{\mathrm{n}}
\end{array}\right]\left[\begin{array}{lll}
\mathrm{l}_{1} & \ldots & \mathrm{l}_{. \mathrm{n}}
\end{array}\right]
$$

Donde li. $(\forall \mathrm{i}=1,2 \ldots \mathrm{n})$ representa las sumas en filas de la inversa de Leontief y $1 . j(\forall \mathrm{j}=1,2 \ldots \mathrm{n})$ las sumas en columnas de dicha matriz, Vrecoge la suma en filas y columnas de los elementos de la inversa de Leontief, es decir, $\mathrm{V}=\sum_{\mathrm{i}} \sum_{\mathrm{j}} \mathrm{l}_{\mathrm{ij}}$.

Esta técnica se encuentra aplicada. entre otros, en los trabajos de Cansino y otros (2013) en el que se aplica la MPM para determinar los sectores clave de la economía española a partir de la matriz de contabilidad social de 2007; el de Aroca y Soza (2013) donde se comparan las estructuras productivas de regiones chilenas; en Ali y otros (2015) se realiza una comparación entre diferentes metodologías, una de las cuales es la MPM, considerando datos de Italia entre los años 1995 y 2011; el trabajo de Viggiano y otros (2017) analiza con esta metodología el sistema productivo brasileño, entre 2005 y 2011; en Beltrán y otros (2017) se estudia a partir de la utilización de la MPM el cambio estructural en México en el período 2003-2012.

\footnotetext{
- Los eslabonamientos hacia delante son calculados a partir de la matriz inversa de Leontief y no de la de Ghosh.
} 


\subsubsection{La complejidad de la estructura productiva}

El transcurso del tiempo hace que las economías vayan aumentando su complejidad al convertirse en economías maduras (Hewings, et al 1988). Una de las técnicas que permite analizar la complejidad es la propuesta por Dietzenbacher et al (2005) y Dietzenbcher y Romero (2007), denominada AveragePropagationLengths (APL), la cual se basa en las expresiones de las inversas de Leontief y Ghosh en términos de series de potencias. Es decir,

$$
\begin{aligned}
& \Delta \mathrm{x}=(\mathrm{I}-\mathrm{A})^{-1} \Delta \mathrm{y}=\left(\mathrm{I}+\mathrm{A}+\mathrm{A}^{2}+\mathrm{A}^{3}+\ldots\right) \Delta \mathrm{y}=\mathrm{L} \Delta \mathrm{y} \\
& \Delta \mathrm{x}^{\prime}=\Delta \mathrm{w}^{\prime}(\mathrm{I}-\mathrm{B})^{-1}=\Delta \mathrm{w}^{\prime}\left(\mathrm{I}+\mathrm{B}+\mathrm{B}^{2}+\mathrm{B}^{3}+\ldots\right)=\Delta \mathrm{w}^{\prime} \mathrm{G}
\end{aligned}
$$

Donde y representa la demanda final,w, los insumos primarios, L esla inversa de Leontiefy $\mathrm{G}$ a la matriz inversa de Ghosh.

Cuanto más largos sean los caminos a través de los cuales se propagan los efectos de un aumento de demanda o de un empuje de los costes, más complejo será un sector o una economía.

Si nos centramos inicialmente en la demanda, los elementos de la matriz $\mathrm{L}=\{$ lij $\}$ tendrán la forma siguiente

$$
l_{i j}=\left\{\begin{array}{c}
a_{i j}+\sum_{k} a_{i k} a_{k j}+\sum_{k} \sum_{s} a_{i k} a_{k s} a_{s j} \ldots \quad \forall i \neq j \\
1+a_{i j}+\sum_{k} a_{i k} a_{k j}+\sum_{k} \sum_{s} a_{i k} a_{k s} a_{s j} \ldots \quad \forall i=j
\end{array}\right.
$$

Suponiendo un incremento en la demanda final, el número medio de pasos requeridos por el sector $\mathrm{j}$ para conseguir afectar al i, será

$$
A P L_{i j}=\left\{\begin{array}{c}
\frac{a_{i j}}{l_{i j}}+2 \frac{\sum_{k} a_{i k} a_{k j}}{l_{i j}}+3 \frac{\sum_{k} \sum_{s} a_{i k} a_{k s} a_{s j}}{l_{i j}}+\cdots \quad \forall i \neq j \\
1+\frac{a_{i j}}{l_{i j}-1}+2 \frac{\sum_{k} a_{i k} a_{k j}}{l_{i j}-1}+3 \frac{\sum_{k} \sum_{s} a_{i k} a_{k s} a_{s j}}{l_{i j}-1}+\cdots \quad \forall i=j
\end{array}\right.
$$

El razonamiento y planteamiento sería análogo si nos referimos a los costes, en este caso se emplearía la matriz inversa de Ghosh (G) para llevar a cabo el análisis.

Por otra parte, el valor de APL coincide al aplicar la inversa de Leontief o la de Ghosh, dadas las propiedades de las matrices involucradas y los vínculos que pueden ser establecidas entre ellas (Dietzenbacher et al, 2005).

El cálculo de APL produce una tabla de doble entrada cuyos elementos pueden ser interpretados tanto en filas como por columnas. En concreto, un elemento APLijindica la longitud de propagación promedio de un "empujón" de costes (dirigido hacia adelante) en el sector i para afectar el valor del output en el sector j. Por otra parte, también proporciona la longitud de propagación promedio de un "pull" de demanda (dirigido hacia atrás) del sector $\mathrm{j}$ a $\mathrm{i}$.

Al considerar las distancias de un sector a cualquier otro en el sistema económico se toman promedios. APL promedio hacia delante puede ser definida como:

$$
\mathrm{APLF}_{\mathrm{i}}=\frac{1}{\mathrm{n}} \sum_{\mathrm{j}} \mathrm{APL}_{\mathrm{ij}}
$$

APL promedio hacia a tras se define de la siguiente manera

$$
\mathrm{APLB}_{\mathrm{j}}=\frac{1}{\mathrm{n}} \sum_{\mathrm{i}} \mathrm{APL}_{\mathrm{ij}}
$$

Donde $\mathrm{n}$ representa el número de sectores considerados en la tabla insumo-producto.

Por último, Romero et al (2009) definen un índice de complejidad de la economía global en los términos siguientes:

$$
\mathrm{CI}=\frac{1}{\mathrm{n}} \sum_{\mathrm{j}} \mathrm{APLF}_{\mathrm{i}}=\frac{1}{\mathrm{n}} \sum_{\mathrm{i}} \mathrm{APLB}_{\mathrm{j}}
$$


Esta metodología ha sido aplicada, entre muchos otros, por autores como Romero et al (2009) a la economía de Chicago; Wang y Sun (2017) al sistema productivo chino olnomata (2014) a los países del Este Asiático.

\subsection{El estudio del cambio estructural. La descomposición estructural}

Dado que estamos analizando la evolución de la economía regional a lo largo de una década, resulta muy adecuado determinar no sólo el cambio estructural que ha experimentado la misma, sino también su descomposición. Siguiendo los trabajos de Fieldman et al (1983) y Sonis et al (1996) en los que se aplica la siguiente descomposición del cambio estructural:

$$
\begin{aligned}
& \Delta \mathrm{x}=\mathrm{x}_{\mathrm{t}}-\mathrm{x}_{0} \\
& \Delta \mathrm{B}=\mathrm{B}_{\mathrm{t}}-\mathrm{B}_{0} \\
& \Delta \mathrm{f}=\mathrm{f}_{\mathrm{t}}-\mathrm{f}_{0}
\end{aligned}
$$

Donde el subíndice t representa el momento final (2016) y 0 el inicial (2007).

Partiendo de la expresión (14) y utilizando las igualdades derivadas del análisis insumo-producto, se tiene:

$$
\Delta \mathrm{x}=\mathrm{x}_{\mathrm{t}}-\mathrm{x}_{0}=\mathrm{B}_{\mathrm{t}} \mathrm{f}_{\mathrm{t}}-\mathrm{B}_{0} \mathrm{f}_{0}
$$

Haciendo $\mathrm{B}_{\mathrm{t}}=\mathrm{B}_{0}+\Delta \mathrm{Byf}_{\mathrm{t}}=\mathrm{f}_{0}+\Delta \mathrm{f}$, la expresión (17) toma la forma

$$
\Delta \mathrm{x}=\mathrm{B}_{0} \Delta \mathrm{f}+\Delta \mathrm{Bf}_{0}+\Delta \mathrm{B} \Delta \mathrm{f}
$$

El primer sumando a la derecha del igual representa el peso que tiene, en el cambio del output, las modificaciones de la demanda final; el segundo término representa la importancia de cambios en la matriz inversa de Leontief (cambios en la tecnología) y el tercer sumando, los cambios debidos a la interacción de $\mathrm{B}$ con la demanda final.

Cada uno de estos componentes del cambio del output, a su vez, puede ser dividido en dos partes: modificacionesdebidas al propio sector o generadas por el resto de sectores. De acuerdo con la notación de Sonis et al (1996), tenemos para un sector genérico i las expresiones que siguen:

$$
\begin{aligned}
& s \Delta x_{i}^{f}=b_{i i} \Delta f_{i} \text { y } n s \Delta x_{i}^{f}=\Delta x_{i}^{f}-s \Delta x_{i}^{f} \\
& s \Delta x_{i}^{B}=\Delta b_{i i} f_{i} \text { y } n s \Delta x_{i}^{B}=\Delta x_{i}^{B}-s \Delta x_{i}^{B} \\
& s \Delta x_{i}^{B f}=\Delta b_{i i} \Delta f_{i} \text { y } n s \Delta x_{i}^{B f}=\Delta x_{i}^{B f}-s \Delta x_{i}^{B f}
\end{aligned}
$$

Donde $s \Delta x_{i}^{f}$ se refiere a los cambios en el output debidos a la demanda final y generados por el propio sector; $\mathrm{ns} \Delta \mathrm{x}_{\mathrm{i}}^{\mathrm{f}}$ denota las modificaciones en el output debidas a la demanda final y generadas por el resto de sectores; $\mathrm{s} \Delta \mathrm{x}_{\mathrm{i}}^{\mathrm{B}}$ representa cambios en el output derivadosdela inversa de Leontief y a variaciones en el propio sector y $n s \Delta x_{i}^{B}$ representan los cambios debidos al resto de sectores; con $s \Delta x_{i}^{B f}$ y ns $\Delta x_{i}^{B f}$ se representan las variaciones en el output debidas a la actuación conjunta deB y f, y generadas por el propio sector y el resto de ellos, respectivamente.

El estudio del cambio estructural y su descomposición ha sido efectuado en trabajos tanto teóricos como aplicados. Dentro de estos últimos podemos referirnos a los de Hahn-De-Castro (2016) para Colombia o Leite (2014) para Brasil, entre otros. 


\section{ESTIMACIÓN DE LA MATRIZ INSUMO-PRODUCTO DE LA REGIÓN DE LOS RÍOS}

Como ya se ha señalado, la metodología aplicada en este trabajo es el análisis insumo-producto, el cual se fundamenta en una MIP. Dado que no existía dicha matriz de la Región de los Ríos para un periodo "actual", ha sido necesario estimarla, en concreto, se ha realizado la estimación correspondiente a 2016.El método aplicado para dicha estimación es la técnica RAS (Stone, 1961) frecuentemente utilizada por sus buenas propiedades y resultados. Dicha técnica plantea un ajuste biproporcional, ya que efectúa una doble corrección por filas y columnas. Para aplicar esta técnicase ha tomado como base la tabla insumo-producto regional de 2007, además ha sido necesarioemplear tres vectores: uno, cuyos elementos son las sumas de la matriz de flujos intermedios en filas, otro vector de sumas de las columnas de la matriz de flujos intermedios y, por último, otro que recoge la producción total de cada sector. Estainformación complementaria ha sido aportada por el Servicio de Impuestos Internos (SII) a 31 de diciembre del $2016^{\circ}$. Una vez determinados estos vectores se ha llevado a cabo una estimación de la matriz de la Región de los Ríos, la cual se encuentra recogida en el ANEXO 1. Asimismo, en el ANEXO 2 se muestra la MIP de 2007 para su consulta.

Con el objetivo de contrastar la adecuación de los resultados de la estimación de la MIP se ha intentado llevar a cabo una comparación con la matriz regional publicada por el Banco Central de Chile, sin embargo, esta última matriz se encuentra desagregada a 12 sectores (a 18 la estimada en este trabajo) y con período de referencia 2013, por lo cual no se ha podido efectuar tal comparación.

Con la finalidad de establecer un marco para el análisis de la estructura productiva, se ha realizado la estimación del PIB regional para el año 2016. Lacual ha tomado como base los resultados derivados en la MIP para la Región, así como los cambios generados por la actual situación económica (véase tabla $\mathrm{N}^{\circ} 1$ ). Cabe destacar que el valor del PIB total representa USD \$2.013 millones de dólares y, en términos per cápita, equivale a USD \$7.984.

\section{ANÁLISIS DE LA ESTRUCTURA PRODUCTIVA DE LA REGIÓN DE LOS RÍOS}

Una vez que se dispone de la MIP para la Región de los Ríos, el paso siguiente será llevar a cabo su análisis, el cual será efectuado desde dos perspectivas diferenciadas: por un lado se realizará un estudio de la economía "real", determinando qué sectores son relevantes para el desenvolvimiento regional y, por otra parte, se llevará a cabo la evaluación de los cambios producidos en la economía regional, a diez años desde su creación y habiendo experimentado esta una descentralización política y administrativa.

\subsection{Caracterización y jerarquía de los sectores productivos regionales}

A partir del cálculo de los eslabonamientos hacia atrás y hacia delante propuestos por Chenery y Watanabe (1958) y Rasmussen (1956) se realizará la caracterización de los sectores. Los resultados se recogen en la tabla 2.

\footnotetext{
- La información complementaria para estimar la MIP de 2016 está accesible en la página web corporativa a través del sitio (www.sii.cl).

- Los valores del PIB proporcionados por el Banco Central de Chile no son coincidentes a nivel de actividad económica con los proporcionados en este trabajo, aunque sí se asemejan si se consideran los montos globales. Los motivos de esta falta de coincidencia se deben fundamentalmente a que los métodos de estimación empleados son diferentes.
} 
Tabla 1

Estimación del Producto Interno Bruto para Región de Los Ríos, por sectores económicos en 2016.

\begin{tabular}{|c|c|c|}
\hline SECTORES & PIB (millones \$) & $\%$ \\
\hline Silvoagropecuario & 302.255 & 15,01 \\
\hline Pesca & 35.620 & 1,77 \\
\hline Minería & 12.054 & 0,60 \\
\hline Alimentos, Bebidas y Tabaco & 209.705 & 10,42 \\
\hline Textil, prendas de vestir y cuero & 1.661 & 0,08 \\
\hline Madera y Muebles & 32.632 & 1,62 \\
\hline Papel e Imprentas & 4.504 & 0,22 \\
\hline Resto industria & 58.738 & 2,92 \\
\hline Electricidad, gas y agua & 24.335 & 1,21 \\
\hline Construcción & 192.919 & 9,58 \\
\hline Comercio, Restaurant y Hoteles & 416.095 & 20,67 \\
\hline Transporte & 108.491 & 5,39 \\
\hline Comunicaciones & 132.268 & 6,57 \\
\hline Servicios Financieros & 112.933 & 5,61 \\
\hline Educación & 124.360 & 6,18 \\
\hline Salud & 45.483 & 2,26 \\
\hline Resto Serv. Pers, P. Viv. y Otros Bs. y Ss. & 114.151 & 5,67 \\
\hline Administración Pública & 84.910 & 4,22 \\
\hline Total PIB Región de Los Ríos & 2.013 .113 & $100 \%$ \\
\hline
\end{tabular}

Fuente: Elaboración propia.

Tabla 2

Clasificación de sectores productivos de la Región de los Ríos

\begin{tabular}{l|llll}
\hline & \multicolumn{3}{l}{ Chenery-Watanabe } & \multicolumn{2}{l}{ Rasmussen } \\
& 2007 & 2016 & 2007 & 2016 \\
\hline Silvoagropecuario & Clave & Clave & Clave & Clave \\
Pesca & Independiente & Impulsor & Independiente & Impulsor \\
Minería & Independiente & Impulsor & Independiente & Impulsor \\
Alimentos, Bebidas y Tabaco & Clave & Clave & Clave & Clave \\
Textil, prendas de vestir y cuero & Impulsor & Impulsor & Impulsor & Impulsor \\
Madera y Muebles & Impulsor & Independiente & Impulsor & Independiente \\
Papel e Imprentas & Impulsor & Impulsor & Impulsor & Impulsor \\
Resto industria & Base & Independiente & Base & Independiente \\
Electricidad, gas y agua & Clave & Independiente & Independiente & Independiente \\
Construcción & Independiente & Independiente & Independiente & Independiente \\
Comercio, Restaurantes y Hoteles & Impulsor & Base & Impulsor & Base \\
Transporte & Clave & Base & Base & Base \\
Comunicaciones & Impulsor & Independiente & Impulsor & Independiente \\
Servicios Financieros & Base & Base & Base & Independiente \\
Educación & Independiente & Independiente & Independiente & Independiente \\
Salud & Independiente & Independiente & Independiente & Independiente \\
Resto Serv. Pers, P. Viv. y Otros Bienes y Servicios & Independiente & Impulsor & Independiente & Independiente \\
Administración Pública & Impulsor & Independiente & Independiente & Independiente \\
\hline Fund E & & &
\end{tabular}


Como puede apreciarse en la tabla anterior, las clasificaciones determinadas para cada año a partir de los coeficentes de Chenery Watanabe y de Rasmussen son bastante similares, ya que en términos generales los efectos indirectos tienen un peso inferior a los directos.

Considerando la información relativa a 2016, podemos señalar que la economía de la Región se fundamenta, según Rasmussen, en sectores clave tales como Silvoagropecuario, Alimentos, Bebidas y Tabaco; impulsores, entre los que se encuentran Pesca, Minería, Textil, prendas de vestir y cuero y Papel e Imprentas y las ramas base Comercio, Restaurantes y Hoteles y Transporte. Si consideramos los indicadores de Chenery y Watanabe, pueden apreciarse dos diferencias (en relación a Rasmussen) en los sectores Servicios Financieros, que son clasificados como base y en Resto de Servicios Personales, determinados como impulsores.

Parece que la economía de la Región se encuentra diversificada, ya que las ramas relevantes están distribuidas en los tres grandes sectores (primario, secundario y terciario).

En lo que se refiere a la situación en 2007, los sectores relevantes tanto por un elevado BL como FL fueron Silvoagropecuario, Alimentos, Bebidas y Tabaco, según Rasmussen y Electricidad, gas y agua y Transporte, además de los dos anteriores, según los coeficientes de Chenery y Watanabe. Dentro de los impulsores podemos señalar Textil, prendas de vestir y cuero y Papel e Imprentas, Madera y Muebles, Comunicaciones y Comercio, Restaurantes y Hoteles. Los sectores Servicios financieros y Resto de la industria son base según ambos indicadores, siendo también base según Rasmussen, el sector del Transporte.

Si efectuamos una comparación entre la estructura de la economía de la Región de los Ríos entre 2007 y 2016, se observa que los sectores Silvoagropecuario, Alimentos, Bebidas y Tabaco, Textil, prendas de vestir y cuero, Papel e Imprentas, Construcción, Educación, Salud, Resto de Servicios Personales y Otros Bienes y Servicios, no han variado de tipología. Sin embargo, algunas otras ramas sí han presentado modificaciones, así por ejemplo, Pesca y Minería que eran independientes en 2007 han pasado a convertirse en impulsoras en 2016; en sentido contrario se ha modificado la tipología del sector Comunicaciones que ha pasado de impulsor a independiente y Resto de Industria fue base de la economía en 2007 e independiente en 2016. Es decir, los sectores Comunicaciones y Resto de Industria han perdido importancia en la economía de la Región, habiéndola ganado Pesca y Minería.

Los resultados obtenidos son coherentes con los datos publicados por el Servicio de Impuestos Internos (SII) en la Estadística de Empresas por Región, Comuna, Rubro y Subrubro (2005-2015), la cual muestra la importancia de los sectores Silvoagropecuario, Alimentos, Bebidas y Tabaco, Comercio, Transporte y Madera y Muebles ${ }^{\circ}$.

Abundando en la evaluación de la relevancia de los sectores, se ha calculado y representado gráficamente la matriz MPM, observándose los siguientes resultados:

\footnotetext{
- Esta encuesta toma como punto de referencia las ventas efectuadas por el sector.
} 
Figura 1

Gráfica de la Matriz MPM de la Región de los Ríos, 2007

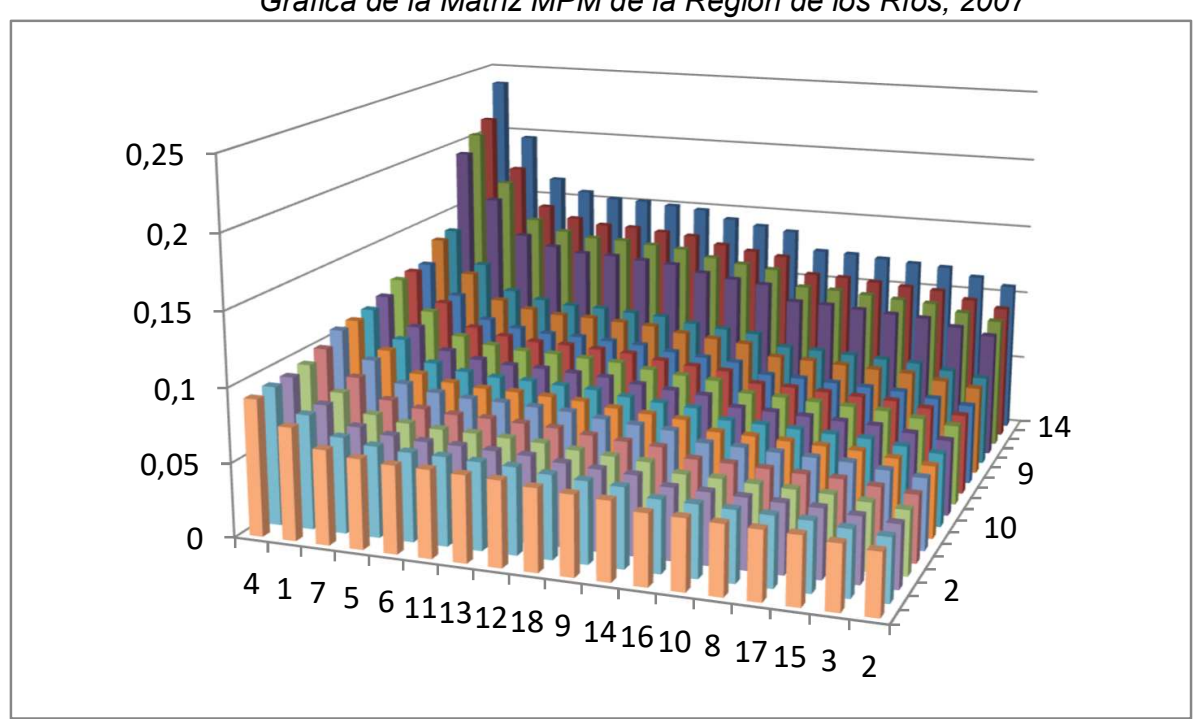

Fuente: Elaboración propia.

A partir del gráfico anterior se pueden establecer ordenaciones entre los diferentes sectores para el año analizado. Puede apreciarse que los sectores con BL más elevado son Alimentos, Bebidas y Tabaco (4), Silvoagropecuario (1), Papel e Imprentas (7), Textil, prendas de vestir y cuero (5) y Madera y Muebles (6) y Comercio, Hoteles y Restaurantes (11). Si consideramos los FL, se puede apreciar que los sectores más relevantes son Servicios Financieros (14), Silvoagropecuario (1), Resto de la Industria (8) y Alimentos, Bebidas y Tabaco (4). Estos resultados son coincidentes con los calculados a partir de los indicadores de Rasmussen.

Si se determina ahora la matriz MPM referida a 2016, los resultados se muestran en el gráfíco N.2. Como puede apreciarse, la estructura productiva mostrada para este año es diferente a la de 2007, esto se debe fundamentalmente al importante crecimiento que ha experimentado el sector de Alimentos, Bebidas y Tabaco.

Desde la óptica de los BL, el sector que destaca del resto, como ya se ha señalado, es Alimentos, Bebidas y Tabaco (4), también podemos señalar Pesca (2), Textil, prendas de vestir y cuero (5), Silvoagropecuario (1), Papel e Imprentas (7) y Minería (3) que guardan semejanza con los determinados para 2006. En lo que se refiere a los FL, las ramas con valores más elevados son Alimentos, Bebidas y Tabaco (4), Silvoagropecuario (1), Comercio, Restaurantes y Hoteles (11), Transporte (12) y Servicios Financieros (14). Los resultados son análogos a los obtenidos mediante el indicador de Rasmussen.

\footnotetext{
- Los sectores recogidos en la gráfica son: 1. Silvoagropecuario; 2. Pesca; Resto Minería; 4. Alimentos, Bebidas y Tabaco; 5. Textil, prendas de vestir y cuero; 6. Madera y Muebles; 7.Papel e imprentas; 8.Resto Industria; 9. Electricidad, gas y agua; 10. Construcción; 11. Comercio, Restaurantes y Hoteles; 12.Transporte; 13. Comunicaciones; 14. Servicios Financieros; 15. Educación; 16. Salud; 17. Resto de Servicios Personales, y Otros bienes y servicios y 18. Administración Pública.
} 
Figura 2

Gráfica de la Matriz MPM de la región de los Ríos, 2016

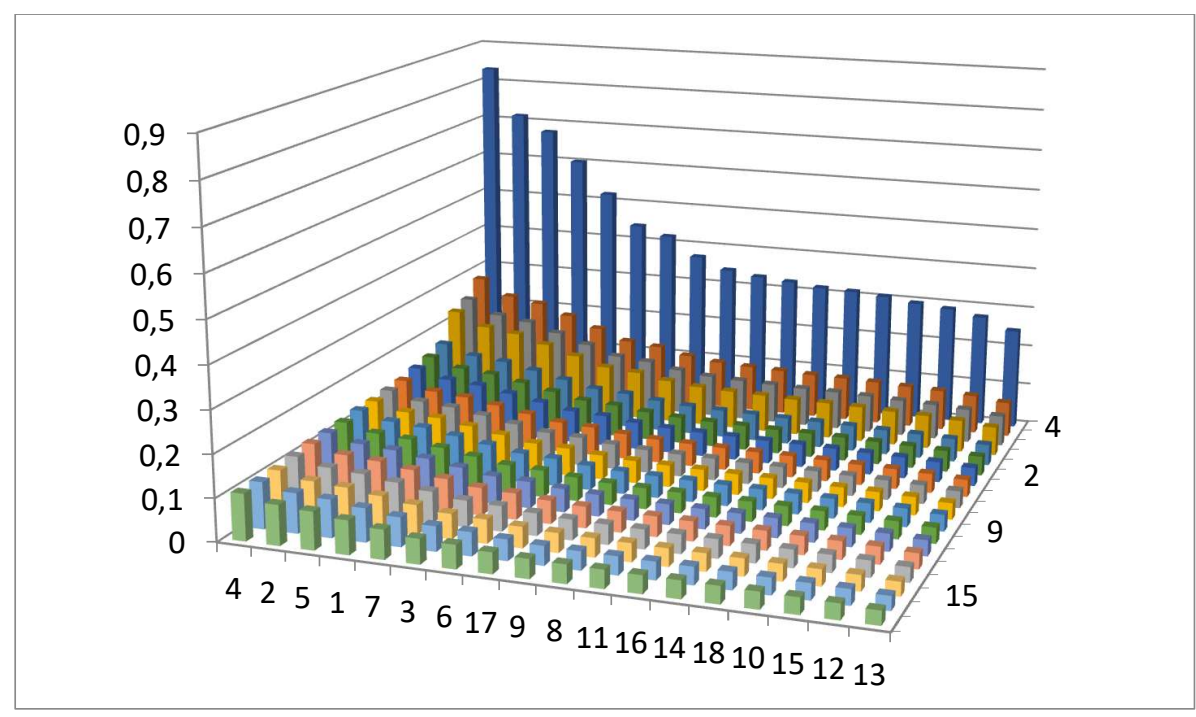

Fuente: Elaboración propia.

A partir de la determinación de la matriz MPM, se pueden comparar las estructuras productivas en los dos momentos temporales considerados. Siguiendo a Guo y Hewings (2001) se analizan los cambios experimentados en las jerarquías de los sectores en relación al valor de sus encadenamientos en ambos años considerados. Los cambios experimentados en los sectores respecto al BL se recogen en la tabla 3.

Como se puede observar, las ramas que han ascendido en la jerarquía determinada a partir del valor del BL son Textil, prendas de vestir y cuero, Electricidad, gas y agua, Resto de industria, Resto de Servicios Personales, y muy especialmente, Minería y Pesca. Estos sectores han cobrado importancia en la economía de la Región de los Ríos, ya que han aumentado su demanda de los bienes y servicios producidos por otras ramas. En el sentido opuesto, los sectores Silvoagropecuario, Papel e imprentas, Madera y muebles, Comercio, Restaurantes y Hoteles, Comunicaciones, Transporte, Administración pública, Servicios Financieros y Construcción han descendido en la jerarquía sectorial, demandando menos bienes y servicios del resto de sectores. Educación y Salud permanecen en la misma posición en ambos períodos considerados.

En lo que respecta a los eslabonamientos hacia delante (FL), si se realiza una comparación de las jerarquías sectoriales en el tiempo, podemos obtener los siguientes resultados: las ramas productivas que han mejorado su posición en la economía de la Región de los Ríos, han sido Alimentación, Bebidas y Tabaco, Comercio, Restaurantes y Hoteles, Transporte, Comunicaciones, Madera y Muebles, Resto de servicios personales, Pesca y Educación. Estos sectores han aumentado, en términos relativos, su oferta de bienes y servicios al resto de la economía. Se puede apreciar que se ha producido un ascenso de sectores de servicios relacionados en mayor o menor medida con el sector turístico (Comercio, Restaurantes y Hoteles, Transporte, Comunicaciones). Por otra parte, el sector pesquero está tomando un mayor peso en la economía, tanto por su BL como por su FL, al haber mejorado su posición en ambas categorías. Lo mismo ocurre con Resto de Servicios Personales.

Servicios Financieros, Resto de Industria, Electricidad, gas y agua, Papel e imprentas, Textil, prendas de vestir y cuero, Construcción y Salud, han disminuido su oferta de productos al resto de la economía. Como puede apreciarse, los sectores de Papel e imprentas y Construcción están perdiendo peso en la economía regional, al ocupar lugares más bajos en la jerarquía, tanto en lo que se refiere a sus encadenamientos hacia delante como hacia atrás.

Las ramas Minería, Silvoagropecuario y Administración Pública no han experimentado cambios significativos y ocupan el mismo lugar en la jerarquía sectorial en ambos años considerados. 
Tabla 3

Comparación de jerarquía de sectores (MPM). BL

\begin{tabular}{llll}
\hline & 2007 & & 2016 \\
\hline 1 & Alimentos, Beidas. y Tabaco & 1 & Alimentos, Bebidas y Tabaco \\
2 & Silvoagropecuario & 2 & Pesca \\
3 & Papel e Imprentas & 3 & Textil, prendas de vestir y cuero \\
4 & Textil, prendas de vestir y cuero & 4 & Silvoagropecuario \\
5 & Madera y Muebles & 5 & Papel e Imprentas \\
6 & Comercio, Restaurantes y Hoteles & 6 & Minería \\
7 & Comunicaciones & 7 & Madera y Muebles \\
8 & Transporte & 8 & Resto Serv. Pers, P. Viv. y Otros Bs. y Ss. \\
9 & Administración Pública & 9 & Electricidad, gas y agua \\
10 & Electricidad, gas y agua & 10 & Resto industria \\
11 & Comunicaciones & 11 & Comercio, Restaurantes y Hoteles \\
12 & Salud & 12 & Salud \\
13 & Construcción & 13 & Servicios Financieros \\
14 & Resto industria & 14 & Administración Pública \\
15 & Resto Serv. Pers, P. Viv. y Otros Bs. y Ss. & 15 & Construcción \\
16 & Educación & 16 & Educación \\
17 & Minería & 17 & Transporte \\
18 & Pesca & 18 & Comunicaciones \\
\hline
\end{tabular}

Fuente: Elaboración propia.

Con el objetivo de contrastar los resultados obtenidos en este análisis se ha realizado una comparación con los datos de la Estadística de Empresas por Región, Comuna, Rubro y Subrubro (2005-2015), apreciándose, en términos generales, conclusiones congruentes. Así, en la anterior estadística señalada también se recoge una pérdida de importancia en el sector Silvoagropecuario, aproximadamente de un 5\%, en relación a su volumen de ventas, aunque se mantiene como uno de los más relevantes en la economía. Por otra parte, el sector pesquero ha visto aumentar sus ventas en el período considerado en un $29 \%$, lo que es coherente con los resultados obtenidos en este artículo. En este mismo sentido, la rama de Alimentación, Bebidas y Tabaco ha experimentado un notable crecimiento en sus ventas de aproximadamente un 109\%; Madera y Muebles también ha crecido en un $28 \%$. Además, podemos señalar el descenso en las ventas de un $75 \%$ que ha experimentado el sector Servicios Financieros. 
Tabla 4

Comparación de jerarquía de sectores (MPM). FL

\begin{tabular}{llll}
\hline & 2007 & \multicolumn{2}{l}{2016} \\
\hline 1 & Servicios Financieros & 1 & Alimentos, Bebidas y Tabaco \\
2 & Silvoagropecuario & 2 & Silvoagropecuario \\
3 & Resto industria & 3 & Comercio, Restaurantes y Hoteles \\
4 & Alimentos, Bebidas y Tabaco & 4 & Transporte \\
5 & Transporte & 5 & Servicios Financieros \\
6 & Electricidad, gas y agua & 6 & Pesca \\
7 & Papel e Imprentas & 7 & Comunicaciones \\
8 & Comercio, Restaurantes y Hoteles & 8 & Resto Serv. Pers, P. Viv. y Otros Bs. y Ss. \\
9 & Comunicaciones & 9 & Resto industria \\
10 & Textil, prendas de vestir y cuero & 10 & Madera y Muebles \\
11 & Construcción & 11 & Electricidad, gas y agua \\
12 & Madera y Muebles & 12 & Papel e Imprentas \\
13 & Resto Serv. Pers, P. Viv. y Otros Bs. y Ss. & 13 & Construcción \\
14 & Minería & 14 & Minería \\
15 & Salud & 15 & Textil, prendas de vestir y cuero \\
16 & Pesca & 16 & Educación \\
17 & Educación & 17 & Salud \\
18 & Administración Pública & 18 & Administración Pública \\
\hline & & & \\
\hline
\end{tabular}

\subsection{La complejidad de la estructura productiva de la Región y su evolución}

A continuación, se va a determinar el nivel de complejidad de la economía de la Región de los Ríos, aplicando la formulación de Dietzenbacher et al (2005), comparando los resultados para 2007 y 2016.

Si consideramos los valores de APLBj en 2007, podemos apreciar que los más elevados (por encima del Índice de complejidad global) corresponden a los sectores Alimentos, Bebidas y Tabaco, Textil, prendas de vestir y cuero, Silvoagropecuario, Pesca, Minería, Comunicaciones y Electricidad, gas y agua, estos son los sectores con mayor longitud media de propagación debida a un aumento en la demanda. Si se observan los APLFi, los sectores con mayor longitud de propagación hacia el resto, generada por un impulso en los costes, son Silvoagropecuario, Pesca, Alimentos, Bebidas y Tabaco, Minería, Madera y Muebles, Educación, Salud y Administración Pública.

Si prestamos atención a la situación en el año 2016, se observa que los valores más elevados de APLBj (por encima del Índice de complejidad global) corresponden a Alimentos, Bebidas y Tabaco, Textil, prendas de vestir y cuero, Silvoagropecuario y Pesca. Los sectores con mayor APLFison Silvoagropecuario, Pesca, Alimentos, Bebidas y Tabaco, Minería, Salud y Administración Pública, es decir, los mismos que en 2007 con la excepción de Madera y Muebles y Educación. Para la mayoría de los sectores salvo Textil (APLFi), Electricidad, gas y agua (APLBj) y Comunicaciones (APLBjy APLFi) que han experimentado pequeñas reducciones, las longitudes medias de propagación han aumentado a lo largo del período considerado, sobre todo en Pesca, Alimentos, Bebidas y Tabaco y Resto de Industria. 
Tabla 5

Complejidad de la economía de la Región de los Ríos

\begin{tabular}{|c|c|c|c|c|c|c|}
\hline \multirow[b]{2}{*}{ Sector } & \multicolumn{2}{|c|}{2007} & \multicolumn{2}{|c|}{2016} & \multicolumn{2}{|c|}{$2007-2016$} \\
\hline & $\mathrm{APLB}_{\mathrm{j}}$ & $\mathrm{APLF}_{\mathrm{i}}$ & $\mathrm{APLB}_{\mathrm{j}}$ & $\mathrm{APLF}_{\mathrm{i}}$ & $\begin{array}{r}\text { Cambio } \\
\text { APLB }(\%) \\
\end{array}$ & $\begin{array}{r}\text { Cambio } \\
\text { APLF }(\%) \\
\end{array}$ \\
\hline Silvoagropecuario & 2,209 & 2,564 & 2,5820 & 3,5718 & 16,89 & 39,30 \\
\hline Pesca & 2,290 & 2,407 & 3,7329 & 3,3706 & 63,04 & 40,03 \\
\hline Minería & 1,995 & 2,113 & 2,0602 & 2,6436 & 3,26 & 25,14 \\
\hline Alimentos, Bebidas y Tabaco & 2,453 & 2,331 & 3,5929 & 3,6680 & 46,46 & 57,38 \\
\hline Textil, prendas de vestir y cuero & 2,323 & 1,820 & 3,5000 & 1,7873 & 50,67 & $-1,82$ \\
\hline Madera y Muebles & 2,030 & 2,008 & 2,2249 & 2,2705 & 9,60 & 13,05 \\
\hline Papel e Imprentas & 1,994 & 1,804 & 2,3357 & 2,1406 & 17,15 & 18,69 \\
\hline Resto industria & 1,827 & 1,669 & 2,8449 & 2,1916 & 55,68 & 31,31 \\
\hline Electricidad, gas y agua & 2,108 & 1,608 & 2,0204 & 1,9526 & $-4,16$ & 21,41 \\
\hline Construcción & 1,936 & 1,808 & 2,1365 & 2,0557 & 10,38 & 13,73 \\
\hline Comercio, Restaurantes y Hoteles & 1,810 & 1,752 & 2,2558 & 1,9252 & 24,65 & 9,88 \\
\hline Transporte & 1,781 & 1,682 & 2,1122 & 1,9171 & 18,60 & 13,98 \\
\hline Comunicaciones & 1,990 & 1,838 & 1,9809 & 1,7519 & $-0,48$ & $-4,67$ \\
\hline Servicios Financieros & 1,724 & 1,559 & 2,2577 & 1,8689 & 30,96 & 19,84 \\
\hline Educación & 1,744 & 2,013 & 1,9614 & 2,2161 & 12,45 & 10,08 \\
\hline $\begin{array}{l}\text { Salud } \\
\text { Resto Serv. Pers, P. Viv. y Otros }\end{array}$ & 1,703 & 2,227 & 2,0336 & 3,1364 & 19,45 & 40,82 \\
\hline Bs. y Ss. & 1,667 & 1,684 & 1,9932 & 1,8945 & 19,58 & 12,49 \\
\hline Administración Pública & 1,771 & 2,467 & 2,0694 & 3,3324 & 16,87 & 35,10 \\
\hline Índice de complejidad global & 1,9 & & & & & \\
\hline
\end{tabular}

Fuente: Elaboración propia.

Si consideramos, por último, el índice de complejidad global de la economía, se observa que la estructura económica de la Región de los Ríos es más compleja en 2016 que en 2007, es decir, la longitud media de propagación global de la economía ha aumentado en un $23.59 \%$, lo que concuerda con la hipótesis propuesta por Hewings et al (1988) de aumento de la complejidad cuando se va haciendo más madura.

\subsection{Descomposición del cambio en el output}

Por último, y si efectuamos la comparación de la evolución del output sectorial producido en la Región de los Ríos descompuesto en sus componentes podemos observar los siguientes resultados: 
Tabla 6

Descomposición del cambio en output por fuentes

\begin{tabular}{|c|c|c|c|c|c|}
\hline Sector & Df & $\mathrm{B}$ & Interacción & Total & $\begin{array}{c}\% \text { sobre } \\
\text { total }\end{array}$ \\
\hline Silvoagropecuario & 534.921 & -13.802 & -42.770 & 478.349 & 21,21 \\
\hline Pesca & 34.880 & 9.569 & 19.340 & 63.788 & 2,83 \\
\hline Minería & 29.241 & -1.616 & -4.763 & 22.862 & 1,01 \\
\hline Alimentos, Bebidas y Tabaco & 166.208 & 164.642 & 311.090 & 641.941 & 28,46 \\
\hline Textil, prendas de vestir y cuero & 5.408 & -1.654 & -3.691 & 0.063 & 0,00 \\
\hline Madera y Muebles & 12.609 & -1.378 & -3.686 & 7.545 & 0,33 \\
\hline Papel e Imprentas & 13.971 & -2.722 & -8.431 & 2.818 & 0,12 \\
\hline Resto industria & 100.067 & -20.671 & -52.207 & 27.190 & 1,21 \\
\hline Electricidad, gas y agua & 36.223 & -6.754 & -22.515 & 6.954 & 0,31 \\
\hline Construcción & 128.372 & -4.132 & -3.158 & 121.081 & 5,37 \\
\hline Comercio, Restaurantes y Hoteles & 317.800 & 26.423 & 95.238 & 439.462 & 19,48 \\
\hline Transporte & 40.649 & 3.826 & 12.068 & 56.543 & 2,51 \\
\hline Comunicaciones & 95.317 & 4.811 & -5.340 & 94.788 & 4,20 \\
\hline Servicios Financieros & 162.084 & -25.542 & -90.673 & 45.869 & 2,03 \\
\hline Educación & 99.071 & -0.228 & -0.427 & 98.416 & 4,36 \\
\hline Salud & 20.046 & -1.445 & -2.766 & 15.835 & 0,70 \\
\hline Resto Serv. Pers, P. Viv. y Otros Bs. y Ss. & 68.197 & 0.822 & 0.973 & 69.992 & 3,10 \\
\hline Administración Pública & 62.102 & -0.044 & -0.012 & 62.045 & 2,75 \\
\hline
\end{tabular}

Como puede apreciarse en la tabla anterior, en todos los sectores se ha producido un aumento en el output, siendo en casi todos ellos el cambio en la demanda final la componente más relevante (a excepción de Alimentos, Bebidas y Tabaco). Este aumento debido a la demanda final ha sido especialmente fuerte en los sectores Silvoagropecuario, Comercio, Restaurantes y Hoteles, Servicios Financieros y Construcción.

Puede observarse que el cambio experimentado por el output de los sectores Pesca, Alimentos, Bebidas y Tabaco, Comercio, Restaurantes y Hoteles, Transporte y Resto de servicios personales presentan todas sus componentes positivas (cambios en la demanda final, en tecnología y sinérgicos), mientras que el resto de ramas consideradas en este estudio presentan cambios en tecnología y sinérgicos con efectos negativos sobre la variación total del output.

Como puede apreciarse en la última columna, los sectores que mayor modificación del output han experimentado en términos porcentuales son Silvoagropecuario; Alimentación, Bebidas y Tabaco; Comercio, Restaurantes y Hoteles. Estos sectores aparecen repetidamente como relevantes para la economía de la Región, según prácticamente todos los indicadores considerados. En este sentido, puede verse el artículo de Ferrada y otros (2014) sobre la competitividad y el desempeño en el sector Silvoagropecuario en el cual se pone de manifiesto la importancia de dicho sector en la Región. Asimismo, en Torres (2010) se señala el peso del sector de Alimentación, Bebidas y Tabaco en el PIB, lo que hace que sea una de las ramasmás relevantes de la economía regional.

Además de la descomposición del cambio en el output por fuentes, también puede ser relevante la consideración de su procedencia, es decir, si ha sido debido a la actividad del propio sector o a la del resto de sectores. En la tabla 7 se detallan los resultados obtenidos. Así, podemos apreciar que el output total de los sectores Silvoagropecuario, Construcción, Comercio, Restaurantes y Hoteles, Comunicación, Educación, Salud y Administración Pública ha aumentado debido a la demanda final que el propio sector ha generado, es decir, son ramas cuyo output ha crecido por el desarrollo de su propia actividad. Por otra parte, las ramas Alimentos, Bebidas y Tabaco, Resto de Industria, Electricidad, Gas y agua y Servicios 
Financieros han experimentado un aumento en su output debido a la demanda final de otros sectores. Por último, en la rama de Alimentos, Bebidas y Tabaco podemos ver la importancia del cambio tecnológico del propio sector en la modificación experimentada por el output.

Tabla 7

Descomposición del cambio en output por fuentes y procedencia

\begin{tabular}{|c|c|c|c|c|c|c|}
\hline \multirow[b]{2}{*}{ Sector } & \multicolumn{3}{|c|}{ Propio sector } & \multicolumn{3}{|c|}{ Resto de sectores } \\
\hline & $\mathrm{DF}$ & B & Interacción & DF & $\mathrm{B}$ & Interacción \\
\hline Silvoagropecuario & 506.777 & -2.707 & -39.565 & 28.144 & -11.095 & -3.206 \\
\hline $\begin{array}{l}\text { Pesca } \\
\text { Minería }\end{array}$ & $\begin{array}{l}33.565 \\
19.546\end{array}$ & $\begin{array}{l}1.481 \\
0.002\end{array}$ & $\begin{array}{l}4.481 \\
0.074\end{array}$ & $\begin{array}{l}1.314 \\
9.695\end{array}$ & $\begin{array}{r}8.088 \\
-1.618\end{array}$ & $\begin{array}{l}14.858 \\
-4.838\end{array}$ \\
\hline $\begin{array}{l}\text { Alimentos, Bebidas y } \\
\text { Tabaco } \\
\text { Textil, prendas de vestir y } \\
\text { cuero }\end{array}$ & 31.306 & 113.628 & 27.365 & 134.902 & 51.014 & 283.725 \\
\hline Madera y Muebles & 1.056 & 1.352 & 0.044 & 11.553 & -2.730 & -3.730 \\
\hline Papel e Imprentas & 3.770 & -0.108 & -0.248 & 10.201 & -2.614 & -8.183 \\
\hline Resto industria & 20.741 & -0.732 & -2.330 & 79.326 & -19.938 & -49.877 \\
\hline Electricidad, gas y agua & 6.992 & -1.101 & -0.635 & 29.231 & -5.653 & -21.880 \\
\hline $\begin{array}{l}\text { Construcción } \\
\text { Comercio, Restaurantes y }\end{array}$ & 116.116 & 0.108 & 0.189 & 12.255 & -4.241 & -3.347 \\
\hline Hoteles & 302.290 & 1.572 & 20.112 & 15.510 & 24.851 & 75.126 \\
\hline Transporte & -4.703 & -1.403 & 0.212 & 45.352 & 5.229 & 11.856 \\
\hline Comunicaciones & 82.742 & -1.532 & -10.241 & 12.574 & 6.343 & 4.901 \\
\hline Servicios Financieros & 34.765 & -2.754 & -3.933 & 127.319 & -22.787 & -86.740 \\
\hline Educación & 98.423 & -0.105 & -0.446 & 0.648 & -0.123 & 0.018 \\
\hline Salud & 17.397 & -0.905 & -0.450 & 2.648 & -0.539 & -2.316 \\
\hline Resto Serv. Personales & 58.058 & 1.161 & 0.984 & 10.139 & -0.339 & -0.011 \\
\hline Administración Pública & 61.883 & 0.000 & -0.001 & 0.219 & -0.044 & -0.011 \\
\hline
\end{tabular}

\section{CONCLUSIONES}

En este artículo se anliza la evolución de la economía de la Región de los Ríos desde su creación hasta la actualidad, 2007-2016., para ello se han empleado diferentes técnicas dentro de la metodología insumoproductoA partir de la aplicación de los indicadores de Chenery y Watanabe y Rasmussen se ha obtenido que en 2016, los sectores claves para la economía regional han sido Silvoagropecuario y Alimentación, bebidas y tabaco. Por otra parte, Comercio, Restaurantes y Hoteles y Transporte son base y Pesca, Minería, Textil, prendas de vestir y cuero y Papel e imprenta se pueden catalogar de impulsores.

A partir de la MPM se han determinado las jerarquías de los sectores y se ha visto cómo han variado a lo largo del período estudiado. Si consideramos los valores de los BL, podemos apreciar que los sectores que ocupan las primeras posiciones en la jerarquía son Alimentación, bebidas y tabaco, Pesca, Textil, prendas de vestir y cuero y Silvoagropecuario. Las ramas Pesca y Textil, prendas de vestir y cuero han mejorado su posición a lo largo del período considerado (especialmente la primera), mientras que Silvoagropecuario ha empeorado y el sector alimentario la ha mantenido. Por lo que se refiere a los FL, los sectores más relevantes son Alimentación, bebidas y tabaco, Silvoagropecuario, Comercio, Restaurantes y Hoteles y Transporte. Todos ellos ascendieron posiciones en su jerarquía, salvo Silvoagropecuario que la mantuvo. 
Del estudio de la complejidad de la economía se ha obtenido que en el año 2016 los valores más elevados de APLBj (por encima del Índice de complejidad global) corresponden a Alimentos, Bebidas y Tabaco, Textil, prendas de vestir y cuero, Silvoagropecuario y Pesca. Los sectores con mayor APLFison Silvoagropecuario, Pesca, Alimentos, Bebidas y Tabaco, Minería, Salud y Administración Pública, presentando notables coincidencias con los resultados de 2007.

Por último, se han analizado las variaciones en el output, resultando que en todos los sectores se ha producido un aumento en el output, siendo en casi todos ellos el cambio en la demanda final la componente más relevante (a excepción de Alimentos, Bebidas y Tabaco). Dicho crecimiento debido a la demanda final ha sido muy importante en las ramas Silvoagropecuaria, Comercio, Restaurantes y Hoteles, Servicios Financieros y Construcción. Los sectores que en términos porcentuales mayor cambio en el output han experimentado han sido Silvoagropecuario, Alimentación, Bebidas y Tabaco, Comercio, Restaurantes y Hoteles. Además, el output de los sectores Silvoagropecuario, Construcción, Comercio, Restaurantes y Hoteles, Comunicación, Educación, Salud y Administración Pública ha aumentado debido a la demanda final que el propio sector ha generado. Por otra parte, las ramas Alimentos, Bebidas y Tabaco, Resto de Industria, Electricidad, Gas y agua y Servicios Financieros han experimentado un aumento en su output debido a la demanda final de otros sectores. Por último, en la rama de Alimentos, Bebidas y Tabaco podemos ver la importancia del cambio tecnológico del propio sector en la modificación experimentada por el output.

\section{REFERENCIAS BIBLIOGRÁFICAS}

ALI, Y., CIASCHINI, M., PRETAROLI, R., y SOCCI, C. (2015).“Measuring the economic landscape of Italy: target efficiency and control effectiveness" en Economia e Politicalndustriale, 42(3), pp. 297-321.

AROCA, P. y SOZA, S. (2013). "Diferencias productivas estructurales entre el Centro y la Periferia: Magallanes y Arica versus el promedio nacional" en Magallania (Punta Arenas), 41(2), pp. 101-118.

BELTRÁN, L. D., DELGADO, M. C., y RİOS, H. (2017). “Análisis multisectorial y de cambio estructural de la economía Mexicana para el periodo 2003-2012" en Revista de Estudios Regionales, (110), pp. 69-97.

CANSINO, J. M., CARDENETE, M. A., RÍOS, M. O., y COLLADO, R. R. (2013)."Análisis de sectores clave de la economía española a partir de la Matriz de Contabilidad Social de España 2007"en Estudios de economía aplicada, 31(2), pp. 18-34.

CHENERY, H. B., y WATANABE, T. (1958). "International comparisons of the structure of production"en Econometrica: Journal of the Econometric Society, 26(4), pp. 487-521.

DIETZENBACHER, E. (1997). "In vindication of the Ghosh model: a reinterpretation as a price model"en Journal of regional science, 37(4), pp. 629-651.

DIETZENBACHER, E., ROMERO LUNA, I., y BOSMA, N. S. (2005).“Using average propagation lengths to identify production chains in the Andalusian economy"en Estudios de Economia Aplicada, 23(2), pp. 405-422.

DIETZENBACHER, E., y ROMERO, I. (2007). "Production chains in an interregional framework: identification by means of average propagation lengths"en International Regional Science Review, 30(4), pp. 362-383.

FERRADA, L. M., CANDIA, J., y PÉREZ, C. (2014). "Competitividad y desempeño económico del sector silvoagropecuario a nivel regional en Chile"en Idesia (Arica), 32(4), pp. 83-94.

FELDMAN, S. J., MCCLAIN, D. \& PALMER, K. (1987). "Sources of structural change in the United States 1963-1978: an input-output perspective"enReview of Economics and Statistics, 69(3), pp. 503-510.

FRANCO, H. y RAMÍREZ, A. (2005). "El modelo Harrod-Domar: implicaciones teóricas y empíricas"en Ecos de Economía. 9(21), pp. 127-151.

FREYTAG, A. y FRICKE, S. (2017). "Sectoral linkages of financial services as channels of economic developmentAn input-output analysis of the Nigerian and Kenyan economies"en Review of development finance, 7(1), pp. 3644.

GHOSH, A. (1958). "Input-output approach in an allocation system". Economica, 25(97), 58-64.

GUO, D., y HEWINGS, G. J. (2001): “Comparative analysis of China's economic structures between 1987 and 1997 : an input-output prospective". DiscussionPapers REAL.

HAHN-DE-CASTRO, L. W. (2016).Un ejercicio de descomposición estructural para Colombia: Documentos de Trabajo Sobre Economía Regional y Urbana; 237.

HEWINGS, G.J.D., SONIS, M. y JENSEN, R.C. (1988): "Fields of influence of technological change in input-output models"enPapers of Regional Science. Assoc. 64(1), pp. 25-36

HIRSCHMAN, A.O. (1958). The Strategy of Economic Development. Yale University Press, New Haven. 
INOMATA, S. (2014). "Trade in value added: concept, development, and an East Asian perspective". EnBaldwin, R, Kawai, M. y Wignaraja, S, (ed.): A World Trade Organization for the 21st Century(pp. 48-70).ReinoUnido: Edward Elgar

INE (2004).Matrices Insumo-productoRegionales, 1996.

KELLY, S., TYLER, P., \& CRAWFORD-BROWN, D. (2016). "Exploring vulnerability and interdependency of UK infrastructure using key-linkages analysis"en Networks and Spatial Economics, 16(3), pp. 865-892.

LEITE, F. P. (2014).Evolução Do Emprego E MudançaEstrutural No Brasil No PrimeiroDecênio Dos Anos 2000: Uma AbordagemPasinettiana: In Anais do XLI Encontro Nacional de Economia [Proceedings of the 41th Brazilian Economics Meeting] (No. 169). ANPEC-Associação Nacional dos Centros de Pósgraduação em Economia.

LEONTIEF, W. (1936)."Quantitative input and output relations in the economic systems of the United States"en The review of economic statistics, pp. 105-125.

MIRANDA, J.C. y RAMOS, C (2007).La matriz insumo-producto para la Región de los Ríos: una tarea pendiente:Workshop Internacional Indicadores para el desarrollo: una oportunidad para mirar a la Región de los Ríos. Valdivia (Chile).

MORRONE, H. (2017). "Which sectors to stimulate first in Brazil? Estimating the sectoral power to pull the economy out of the recession"en Investigacióneconómica, 76(302), pp. 55-75.

OOSTERHAVEN, J. (1988). "On the plausibility of the supply-driven input-output model"enJournal of Regional Science, 28(2), pp. 203-217.

OOSTERHAVEN, J. (1996). "Leontief versus Ghoshian price and quantity models"enSouthern Economic Journal, 62(3), pp. 750-759.

OOSTERHAVEN, J. (2012). "Adding supply-driven consumption makes the Ghosh model even more implausible"enEconomic Systems Research, 24(1), pp. 101-111.

PINO, O. y ILLANES, W. (2002).Análisis exploratorio de los coeficientes de Rasmussen para la economía regional, mediante la utilización de las tablas input- output para la economía chilena, base 1996;Encuentro Nacional de Escuelas y Facultades de Administración y Economía (ENEFA), Universidad de Talca, Chile.

POLO, C. y VALLE, E (2007). "Un análisis estructural de la economía balear" en Estadística Española, 49(165), pp. 227-257.

RAMIIREZ, J. C. y SILVA, I. (2008). "Globalización y desarrollo regional: evolución económica de las regiones chilenas, 1990-2002" enRevista de la Cepal, 95, pp. 103-124.

RAMOS, J. L., OTERO, P., LUIS, J., y ARRIETA BARCASNEGRAS, A. (2017). "Análisis insumo-producto y la inversión pública: una aplicación para el Caribe colombiano”. En Cuadernos de Economía, 36(70), pp. 137-167.

RASMUSSEN, P.N. (1956).Studies in IntersectoralRelation. North Holland.

REYES, R. Y MIRANDA, J. C. (1998).La matriz insumo-producto de Valdivia 1994, Propuesta metodológica para el análisis de las relaciones productivas de áreas menores, Ed. REYMI.

ROMERO, I., DIETZENBACHER, E., y HEWINGS, G. J. (2009). "Fragmentation and complexity: analyzing structural change in the Chicago regional economy"en Revista de economíamundial, 23, pp. 263-282.

ROSE, A., y ALLISON, T. (1989). "On the plausibility of the supply-driven input-output model: Empirical evidence on joint stability"en Journal of regional science, 29(3), pp. 451-458.

SII (2016).Estadística de Empresas por Región, Comuna, Rubro y Subrubro (2005-2015).

SONIS, M. y HEWINGS, G. (1999). "Economic Landscapes: Multiplier Product Matrix Analysis for Multirregional InputOutput Systems"enHitotsubashi Journal of Economics, 40, pp.59-74.

SONIS, M., HEWINGS, G. J., y GUO, J. (1996). "Sources of structural change in input-output systems: a field of influence approach"en Economic Systems Research, 8(1), pp. 15-32.

SONIS, M, HEWINGS, G.J y MIYAZAWA, K. (1997). "Synergetic Interactions within the pair-wise Hierarchy of Economic Linkages Sub-Systems"enHitotsubashi Journal of Economics,38, pp. 183-199.

STONE, R. (1961). Input-output and national accounts; Organization for European Economic Co-operation.

THAKUR, S. K. (2011). "Fundamental economic structure and structural change in regional economies: a methodological approach"enRégionetDéveloppement, 33, pp. 9-38.

THAKUR, S. K., y ALVAYAY, J. R. (2012)."Identification of regional fundamental economic structure (FES) of Chilean economy: A field of influence approach"enStructural Change and Economic Dynamics, 23(1), pp. 92-107.

TORRES, S. R. (2010).Capital Humano para la industria alimentaria de la Región de Los Ríos. Memoria de Tesis presentada en la Universidad Austral

VIGGIANO, L., PEROBELLI, F. S., CAMPOY-MUÑOZ, P., y CARDENETE, M. A. (2017). "Análise do Sistema Produtivo Brasileiro em 2005 e 2011 a Partir de Matrizes de Contabilidade Social" en Revista Portuguesa de EstudosRegionais, 45, pp. 7-25.

WANG, Z., y SUN, J. (2017)."Analysis of interregional industry linkage and economic distance in China: evidence from the mining industry" enApplied Economics, 49(6), pp. 606-617. 
ANEXO 1

MATRIZ INSUMO-PRODUCTO DE LA REGIÓN DE LOS RÍOS 2007

\begin{tabular}{|c|c|c|c|c|c|c|c|c|c|c|c|c|c|c|c|c|c|c|}
\hline SECTORES & 1. & 2. & 3. & 4. & 5. & 6. & 7. & 8. & 9. & 10. & 11. & 12. & 13. & 14. & 15. & 16. & 17. & 18. \\
\hline 1. Silvoagropecuario & 17538 & 1 & 29 & 48506 & 12 & 4126 & 58 & 34 & 0 & 67 & 221 & 6 & 0 & 10 & 147 & 15 & 141 & \\
\hline 2. Pesca & 4 & 31 & 0 & 847 & 0 & 0 & 0 & 77 & 0 & 0 & 6 & 0 & 0 & 2 & 2 & 0 & 4 & \\
\hline 3. Minería & 962 & 1 & 2 & 289 & 4 & 119 & 71 & 269 & 11 & 1662 & 2 & 0 & 0 & 27 & 2 & 0 & 12 & \\
\hline 4. Alimentos, Beb. y Tabaco & 16269 & 282 & 0 & 33737 & 235 & 11 & 53 & 2 & 4 & 7 & 1129 & 15 & 1 & 485 & 175 & 176 & 264 & \\
\hline 5. Textil, pren. de vestir y cuero & 63 & 4 & 3 & 77 & 485 & 77 & 20 & 1 & 7 & 261 & 49 & 269 & 8 & 425 & 16 & 97 & 129 & \\
\hline 6. Madera y Muebles & 302 & 2 & 0 & 94 & 0 & 3038 & 1 & 23 & 6 & 3699 & 178 & 50 & 2 & 150 & 8 & 19 & 96 & \\
\hline 7. Papel e Imprentas & 35 & 1 & 4 & 566 & 3 & 279 & 904 & 23 & 54 & 88 & 111 & 423 & 306 & 665 & 338 & 132 & 250 & \\
\hline 8. Resto industria & 3520 & 49 & 79 & 741 & 156 & 605 & 654 & 3709 & 1473 & 3039 & 2467 & 6493 & 569 & 971 & 382 & 639 & 802 & 225 \\
\hline 9. Electricidad, gas y agua & 1317 & 4 & 78 & 1205 & 27 & 732 & 252 & 137 & 2105 & 267 & 815 & 616 & 143 & 706 & 215 & 235 & 416 & 83 \\
\hline 10. Construcción & 9 & 2 & 0 & 307 & 6 & 93 & 10 & 31 & 351 & 41 & 139 & 109 & 175 & 791 & 112 & 87 & 5439 & \\
\hline 11. Comercio, Restaurant y Hotele & 847 & 10 & 5 & 573 & 6 & 57 & 17 & 135 & 33 & 129 & 1024 & 2833 & 596 & 1160 & 158 & 869 & 528 & \\
\hline 12. Transporte & 2472 & 43 & 6 & 4025 & 21 & 1653 & 240 & 239 & 92 & 276 & 1526 & 2018 & 276 & 1685 & 80 & 215 & 312 & \\
\hline 13. Comunicaciones & 208 & 4 & 3 & 242 & 9 & 145 & 8 & 47 & 106 & 107 & 318 & 918 & 2093 & 1506 & 132 & 431 & 183 & \\
\hline 14. Servicios Financieros & 5492 & 62 & 144 & 7462 & 91 & 1072 & 296 & 742 & 2052 & 3218 & 3231 & 2395 & 1964 & 7104 & 1122 & 1927 & 1513 & 273 \\
\hline 15. Educación & 3 & 0 & 0 & 0 & 0 & 0 & 0 & 0 & 3 & 12 & 1 & 10 & 16 & 72 & 109 & 270 & 46 & \\
\hline 16. Salud & 371 & 0 & 0 & 0 & 0 & 0 & 0 & 0 & 0 & 0 & 4 & 33 & 33 & 20 & 3 & 933 & 89 & \\
\hline 17. Resto Serv. Pers, P. Viv. y Otro & 1 & 5 & 25 & 781 & 18 & 347 & 24 & 128 & 12 & 35 & 200 & 9 & 88 & 1103 & 56 & 123 & 693 & \\
\hline $\begin{array}{l}\text { 18. Administración Pública } \\
\end{array}$ & 29 & 0 & 0 & 0 & 0 & 0 & 0 & 0 & 0 & 1 & 1 & 53 & 0 & 0 & 0 & 3 & 0 & \\
\hline Compras intermedias & 49441 & 501 & 378 & 99450 & 1073 & 12355 & 2608 & 5598 & 6308 & 12910 & 11422 & 16249 & 6269 & 16881 & 3058 & 6171 & 10917 & 632 \\
\hline Valor Añadido & 47297 & 11535 & 3552 & 43571 & 2045 & 25174 & 3206 & 26725 & 14690 & 61506 & 20503 & 28639 & 11223 & 47077 & 20704 & 29458 & 61372 & \\
\hline Valor Bruto Producción & 96737 & 12037 & 3930 & 143022 & 3118 & 37529 & 5814 & 32322 & 20998 & 74416 & 31926 & 44888 & 17492 & 63958 & 23762 & 35629 & 72289 & \\
\hline
\end{tabular}

$\begin{array}{llll}96737 & 12037 & 3930 & 143022\end{array}$ 


\section{ANEXO 2}

MATRIZ DE INSUMO PRODUCTO DE LA REGIÓN DE LOS RIOS 2016

(En millones de pesos del año 2016)

\begin{tabular}{|c|c|c|c|c|c|c|c|c|c|c|c|c|c|c|c|c|c|c|c|c|}
\hline SECTORES & 1 & 2 & 3 & 4 & 5 & 6 & 7 & 8 & 9 & 10 & 11 & 12 & 13 & 14 & 15 & 16 & 17 & 18 & $\begin{array}{l}\text { DEMANDA } \\
\text { FINAL }\end{array}$ & VBP \\
\hline 1. Silvoagropecuario & 51.390 & 31 & 1.227 & 112.438 & 13 & 6.470 & 234 & 177 & 112 & 0 & 173 & 0 & 9 & 196 & 5 & 0 & 23 & 481 & 402.108 & 575.086 \\
\hline 2. Pesca & 65 & 6.766 & 0 & 19.337 & 0 & 0 & 0 & 4.958 & 0 & 0 & 0 & 0 & 0 & 48 & 0 & 0 & 36 & 79 & 44.536 & 75.825 \\
\hline 3. Resto Minería & 4.229 & 5 & 24 & 246 & 2 & 67 & 104 & 0 & 1.839 & 104 & 17 & 19 & 82 & 1 & 0 & 0 & 23 & 2 & 20.027 & 26.791 \\
\hline 4. Alimentos, Beb. y Tabaco & 172.015 & 35.757 & 0 & 446.801 & 1.443 & 90 & 1.214 & 0 & 238 & 0 & 0 & 106 & 5 & 5.713 & 68 & 6 & 6.511 & 3.264 & 111.732 & 784.962 \\
\hline 5. Textil, pren. de vestir y cuero & 60 & 61 & 49 & 59 & 173 & 36 & 26 & 0 & 6 & 0 & 0 & 11 & 16 & 14 & 88 & 4 & 486 & 17 & 2.074 & 3.181 \\
\hline 6. Madera y Muebles & 3.387 & 43 & 0 & 257 & 1 & 5.279 & 5 & 19 & 72 & 2.171 & 1.720 & 30 & 585 & 186 & 47 & 2 & 414 & 32 & 30.822 & 45.074 \\
\hline 7. Papel e Imprentas & 120 & 30 & 29 & 913 & 0 & 87 & 838 & 8 & 42 & 26 & 0 & 40 & 2 & 30 & 85 & 85 & 1.453 & 281 & 4.563 & 8.632 \\
\hline 8. Resto industria & 4.075 & 47 & 332 & 736 & 6 & 83 & 96 & 130 & 491 & 2.812 & 25.458 & 251 & 117 & 83 & 339 & 27 & 339 & 45 & 24.042 & 59.512 \\
\hline 9. Electricidad, gas y agua & 1.761 & 28 & 939 & 789 & 8 & 296 & 286 & 97 & 227 & 634 & 1.857 & 2.791 & 10 & 204 & 141 & 53 & 470 & 198 & 17.164 & 27.952 \\
\hline 10. Construcción & 233 & 49 & 0 & 837 & 7 & 156 & 45 & 1 & 141 & 450 & 6.255 & 1.933 & 6 & 144 & 104 & 270 & 2.184 & 431 & 182.252 & 195.497 \\
\hline 11. Comercio, Restaurant y Hoteles & 55.504 & 1.309 & 1.190 & 7.514 & 36 & 464 & 387 & 3.323 & 1.004 & 2.323 & 43.521 & 879 & 98 & 5.126 & 12.996 & 4.433 & 15.426 & 2.925 & 312.930 & 471.387 \\
\hline 12. Transporte & 15.587 & 1.061 & 10.368 & 10.479 & 26 & 3.137 & 1.084 & 656 & 1.074 & 5.494 & 18.234 & 485 & 42 & 1.617 & 1.840 & 408 & 4.452 & 294 & 25.093 & 101.431 \\
\hline 13. Comunicaciones & 2.903 & 163 & 238 & 947 & 16 & 348 & 55 & 124 & 458 & 2.143 & 5.456 & 834 & 24 & 474 & 1.252 & 6.843 & 5.955 & 727 & 83.319 & 112.280 \\
\hline 14. Servicios Financieros & 9.241 & 399 & 873 & 4.995 & 28 & 484 & 343 & 145 & 646 & 16.680 & 13.081 & 2.781 & 125 & 852 & 561 & 899 & 4.826 & 1.060 & 51.807 & 109.827 \\
\hline 15. Educación & 11 & 0 & 0 & 0 & 0 & 0 & 0 & 0 & 0 & 0 & 0 & 25 & 3 & 2 & 14 & 38 & 303 & 638 & 121.144 & 122.178 \\
\hline 16. Salud & 355 & 0 & 0 & 0 & 0 & 0 & 0 & 0 & 0 & 0 & 0 & 0 & 0 & 2 & 14 & 22 & 25 & 5 & 51.041 & 51.464 \\
\hline 17. Resto Serv. Pers, P. Viv. y Otros & 17 & 142 & 1.392 & 2.393 & 26 & 656 & 130 & 515 & 1.152 & 543 & 5.955 & 77 & 6 & 233 & 9 & 153 & 3.429 & 242 & 125.208 & 142.281 \\
\hline 18. Administración Pública & 137 & 0 & 0 & 0 & 0 & 0 & 0 & 0 & 0 & 0 & 0 & 0 & 0 & 2 & 111 & 0 & 0 & 0 & 81.827 & 82.078 \\
\hline Compras Intermedias & 321.091 & 45.892 & 16.662 & 608.740 & 1.785 & 17.652 & 4.847 & 10.153 & 7.502 & 33.380 & 121.727 & 10.262 & 1.130 & 14.925 & 17.674 & 13.243 & 46.355 & 10.724 & 1.691 .692 & 2.995 .438 \\
\hline VALOR AGREGADO & 253.996 & 29.933 & 10.130 & 176.222 & 1.396 & 27.422 & 3.784 & 49.359 & 20.450 & 162.117 & 349.660 & 91.169 & 111.149 & 94.902 & 104.504 & 38.221 & 95.926 & 71.353 & & \\
\hline Valor Bruto de la Producción (VBP) & 575.086 & 75.825 & 26.791 & 784.962 & 3.181 & 45.074 & 8.632 & 59.512 & 27.952 & 195.497 & 471.387 & 101.431 & 112.280 & 109.827 & 122.178 & 51.464 & 142.281 & 82.078 & & \\
\hline Empleo & 25.145 & 1.040 & 884 & 5.275 & 3.609 & 1.705 & 246 & 2.350 & 449 & 12.738 & 13.287 & 3.878 & 1.325 & 316 & 7.839 & 5.390 & 6.920 & 6.982 & & \\
\hline PIR & 3 & 520 & 054 & 209705 & 1661 & 32637 & 4504 & 738 & 24335 & 9919 & 46095 & 891 & 268 & 933 & 4360 & 483 & 4151 & 8401 & & \\
\hline
\end{tabular}

